QCD Evolution Workshop 2014

International Journal of Modern Physics: Conference Series

Vol. 37 (2015) 1560019 (8 pages)

(C) The Author

DOI: $10.1142 / \mathrm{S} 2010194515600198$

\title{
Jefferson Lab 12 GeV Science Program*
}

\author{
R. D. McKeown \\ Jefferson Lab, Newport News, VA 23606, USA \\ Department of Physics, College of William and Mary \\ Williamsburg, VA 23187, USA \\ bmck@jlab.org
}

Published 25 February 2015

\begin{abstract}
The Continuous Electron Beam Accelerator Facility (CEBAF) and associated experimental equipment at Jefferson Lab are presently being upgraded, which will enable a new experimental program with substantial discovery potential to address important topics in nuclear, hadronic, and electroweak physics. Further in the future, it is envisioned that the Laboratory will evolve into an electron-ion colliding beam facility.
\end{abstract}

Keywords: Electron scattering; photoproduction; hadronic structure.

\section{Introduction}

The CEBAF facility at Jefferson Laboratory has operated since 1995, with high-duty factor (continuous) beams of up to $6 \mathrm{GeV}$ electrons incident on three experimental halls (denoted A, B, and C), each with a unique set of experimental equipment. Advanced GaAs photoemission sources have been developed, providing high quality polarized beam with polarizations up to $85 \%$ at beam currents up to $180 \mu \mathrm{A}$. The facility serves an international scientific user community of over 1300 scientists, and 178 experiments have been completed to date. The first decade of scientific results from Jefferson Lab has been summarized in a comprehensive review. ${ }^{1}$ Based on this remarkable record of scientific productivity and the prospects for further scientific advances in this field, a major upgrade of CEBAF to $12 \mathrm{GeV}$ electron beam energy along with substantial new experimental equipment has been planned.

\footnotetext{
*Authored by Jefferson Science Associates, LLC under U.S. DOE Contract No. DE-AC0506OR23177. The U.S. Government retains a non-exclusive, paid-up, irrevocable, world-wide license to publish or reproduce this manuscript for U.S. Government purposes.

This is an Open Access article published by World Scientific Publishing Company. It is distributed under the terms of the Creative Commons Attribution 3.0 (CC-BY) License. Further distribution of this work is permitted, provided the original work is properly cited.
} 


\section{The $12 \mathrm{GeV}$ Upgrade Project}

The upgrade of CEBAF and associated experimental equipment at Jefferson Lab is well underway, with completion expected in 2017. The upgraded facility is schematically shown in Fig. 1. Electron beams up to $11 \mathrm{GeV}$ will be available for experiments in the existing Halls $\mathrm{A}, \mathrm{B}$ and $\mathrm{C}$, and a $12 \mathrm{GeV}$ beam can be delivered to a new experimental Hall D to generate a $9 \mathrm{GeV}$ tagged photon beam, enabling a powerful program of meson spectroscopy. The facility will capable of delivering beam to any 3 of the 4 halls simultaneously, and the ability to deliver beam to all 4 halls at lower duty factor is also anticipated.

The accelerator component of the upgrade project is essentially complete, and commissioning is now underway. In early 2014, electrons were transmitted around around the CEBAF accelerator and achieved full upgrade-energy acceleration of 2.2 $\mathrm{GeV}$ in one pass. The CEBAF operators then delivered beam into a target in Hall A, recording the first data of the $12 \mathrm{GeV}$ era. The operations staff then tuned up a 3pass beam, resulting in $6.11 \mathrm{GeV}$ electrons at 2 nanoAmps average current for more than an hour. On May 3, 2014 the first 5.5 pass beam, with energy of $6.18 \mathrm{GeV}$, was delivered to the front section of the beamline to Hall D, thus demonstrating that all 5.5 passes of the accelerator were functional. Subsequently, the machine delivered its highest-energy beams ever, $10.5 \mathrm{GeV}$, through the entire accelerator and into the Hall D Tagger Facility, which converts CEBAF's electron beam into photons that will be used for experiments in Hall D. Beam commissioning will continue in the Fall of 2014 with the aim of establishing $12 \mathrm{GeV}$ beam energy and delivering simultaneous beam to Hall A and D.

The GlueX experimental apparatus in Hall D is almost complete and will be ready for commissioning in fall 2014. CLAS12 in Hall B and Super High Momentum Spectrometer (SHMS)in Hall C are still in the construction phase. The detector

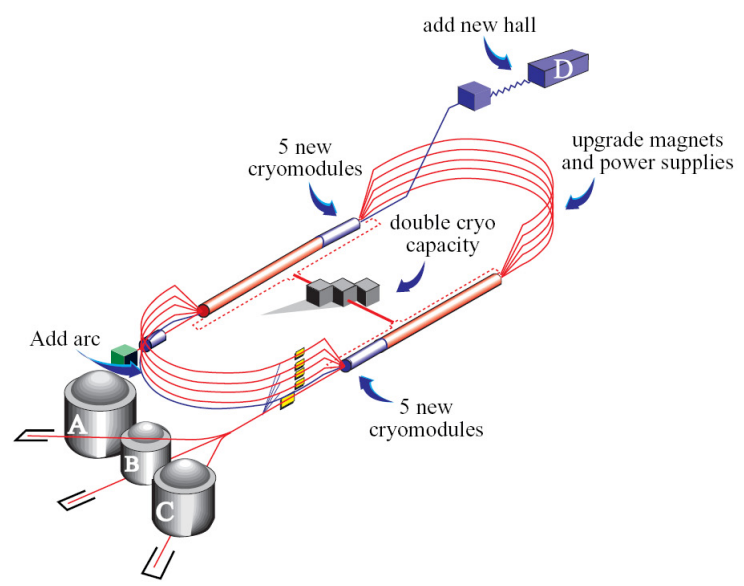

Fig. 1. Jefferson Lab $12 \mathrm{GeV}$ upgrade concept. 
construction is well advanced, while the superconducting magnets for these projects are still under construction at the vendors. Current projections are that CLAS12 and the SHMS spectrometer will be ready for beam commissioning in summer 2016.

\section{The $12 \mathrm{GeV}$ Science Program}

An extensive physics program for the upgraded CEBAF has been developed over the last decade in collaboration with the user community and with the guidance of the Program Advisory Committee. 70 experiments are already approved, and several are conditionally approved. This program will likely require at least a decade to execute.

The major science questions to be addressed with the upgraded facility include:

- What is the role of gluonic excitations in the spectroscopy of light mesons? Can these excitations elucidate the origin of quark confinement?

- Where is the missing spin in the nucleon? Is there a significant contribution from valence quark orbital angular momentum?

- Can we reveal a novel landscape of nucleon substructure through measurements of new multidimensional distribution functions?

- What is the relation between short-range N-N correlations and the partonic structure of nuclei?

- Can we discover evidence for physics beyond the standard model of particle physics?

A detailed discussion of the science opportunities at the upgraded facility has recently been published. ${ }^{2}$ Here we only briefly present some highlights.

\subsection{Meson Spectroscopy}

The excitation of the gluonic field in a quark-antiquark system can lead to mesonic states with exotic quantum numbers $\left(J^{P C}=0^{+-}, 1^{-+}, 2^{+-}\right)$that are not allowed for states with only quark-antiquark degrees of freedom. Such states and their properties have recently been studied in detail using lattice QCD methods. ${ }^{3,4}$ Chiral extrapolation of these lattice calculations to the physical pion mass predict that the exotic mesons should indeed be present in the mass range $2-2.5 \mathrm{GeV}$ that can be studied with the new GlueX experiment sited in Hall D. The main goal of the GlueX experiment is to search for these exotic mesons produced via photoproduction on the nucleon. Linearly polarized photons will be produced upstream of Hall D by the coherent bremmstrahlung process using a thin ( $\sim 20$ micron $)$ diamond wafer. The scattered electrons from 8.5-9 GeV bremmstrahlung photons will be dispersed by a tagger magnet and detected with scintillator detectors, yielding a tagged photon resolution of $0.2 \%$ with fluxes expected to reach $10^{8} / \mathrm{s}$. 


\subsection{Nucleon Structure}

The historic discovery of quarks in deep inelastic scattering at SLAC in the 1960's led to the description of the nucleon in terms of the one-dimensional parton distribution functions (PDF). Four parity conserving structure functions of the momentum fraction $x\left(f_{1}, f_{2}, g_{1}\right.$, and $\left.g_{2}\right)$ are experimentally accessible and have been studied in great detail over the last four decades. However, during the last 15 years it has been realized that these one dimensional distributions are not sufficient to describe some essential physics needed to provide a complete picture of the nucleon. Orbital angular momentum of partons is one important example of a degree of freedom that is not exhibited in the standard 1-D PDFs. Recent experimental and theoretical studies have revealed a more complete description of the partonic structure of the nucleon that is realizable through new multi-dimensional distributions: Generalized Parton Distributions (GPD) and Transverse Momentum Dependent (TMD) distributions.

GPDs contain information on the correlation between the quark/gluon transverse position in the nucleon and the longitudinal momentum fraction $x$. They are accessed in exclusive scattering processes at large $Q^{2}$ : deeply virtual Compton scattering (DVCS) and deep virtual meson production (DVMP). The GPDs offer a path to a full 3-dimensional exploration of nucleon structure, in transverse position and longitudinal momentum space, enabling spatial tomography of the nucleon. The new CLAS12 apparatus being constructed for Hall B as part of the $12 \mathrm{GeV}$ upgrade is particularly well-suited to study these processes.

Transverse momentum dependent distributions (TMDs) contain information on the quark/gluon transverse motion in a nucleon, and on the correlations between the transverse momentum of the quark and the quark/nucleon spins. The TMDs offer a unique opportunity for a momentum tomography of the nucleon, and can be measured in Semi-Inclusive Deep Inelastic Scattering (SIDIS), in which the nucleon is no longer intact and one of the outgoing hadrons is detected. SIDIS will be studied in Hall C with high resolution spectrometers, in Hall B with CLAS12, and in Hall A with the new Super Bigbite Spectrometer (SBS). A major new capability would become available for SIDIS through construction of a new detector system: the Solenoidal Large Intensity Device (SoLID) to be sited in Hall A. This future project would offer exceptional capability for mapping out the TMDs with the 12 GeV beam at JLab.

\section{Quarks in Nuclei}

The Jefferson Lab 12 GeV Upgrade will enable study of the QCD structure of nuclei as well as the use of the nucleus as a laboratory to study QCD. These studies will address of a number of the most fundamental questions in modern nuclear physics.

The nucleon-nucleon (NN) relative wave function at short distances is fundamental to the origin of the nuclear force and to the properties of nuclei. It is not known if the NN system can be described only in terms of nucleons and mesons, 
or whether quarks and gluons are necessary for its description. Recent studies indicate that modification of the nuclear parton distributions, or the "EMC effec", is related to short-range NN correlations in nuclei. ${ }^{5}$ Further experimental studies to explore these important issues will be possible with the $12 \mathrm{GeV}$ CEBAF and new experimental equipment.

QCD also predicts the existence of novel phenomena in nuclear physics. The nuclear medium provides mechanisms for filtering quantum states and studying their spacetime evolution. Hadronization of a struck quark in different nuclei offers a unique method for studying this process. For example, the formation of small color singlet configurations leads to the novel process known as color transparency. The increased kinematic range of the $12 \mathrm{GeV}$ CEBAF will offer new opportunities to study these and other related topics.

Recently, a new method to determine the neutron radius of a heavy nucleus like ${ }^{208} \mathrm{~Pb}$ was pioneered by the PREX experiment. ${ }^{6}$ Precise measurements of the charge distribution of nuclei in elastic electron scattering provide stringent constraints on the distribution of protons in nuclei. The distribution of neutrons is quite important for predicting the properties of neutron stars but is much more difficult to determine experimentally. Since the weak charge of the neutron is -1 , whereas the weak charge of the proton is $1-4 \sin ^{2} \theta_{W} \ll 1$, the measurement of parity violating asymmetries in elastic electron scattering from nuclei is sensitive to the neutron distribution and can be used to constrain the neutron radius. Future studies with higher precision are planned for the Jefferson Lab $12 \mathrm{GeV}$ program.

\section{Beyond the Standard Model}

New opportunities to search for new physics beyond the Standard Model will become possible at Jefferson Lab in the $12 \mathrm{GeV}$ era. Very precise measurements of parity violating asymmetries to study the strange form factors in elastic electron-proton scattering have demonstrated that this technique has substantial potential for precision tests of the Standard Model. The strength of the neutral weak interaction is parameterized in the standard model by the weak mixing angle $\theta_{W}$, which is very precisely determined at the $Z$ boson mass by $e^{+}-e^{-}$collider experiments. The two best measurements can be combined to yield the average value $\sin ^{2} \theta_{W}=0.23116 \pm 0.00013$ [7]. Radiative corrections due to standard model physics produce a "running" of this coupling to $\sin ^{2} \theta_{W}=0.2388$ at $Q^{2}=0$. New physics beyond the standard model at high mass (larger than $M_{Z}$ ) could modify these radiative corrections, leading to a different value of $\sin ^{2} \theta_{W}$ at $Q^{2}=0$. Therefore, precise measurements of the neutral weak interaction at low $Q^{2} \ll M_{Z}^{2}$ can reveal the presence of particles and forces not present in the standard model.

An experiment to study parity-violation in elastic electron-proton scattering at low $Q^{2}, Q_{\text {weak }}$, was recently completed in Hall C at Jefferson Lab. ${ }^{8}$ The data from this experiment are currently under analysis. There are also 2 new proposals to perform parity violation measurements at the upgraded CEBAF. One would use 
the proposed solenoidal magnetic spectrometer system (SOLID) to study parityviolating deep inelastic scattering. ${ }^{9}$ The other proposal is to study parity-violating Møller scattering ${ }^{10}$ utilizing a novel dedicated toroidal spectrometer. Both experiments require construction of substantial new experimental equipment (beyond the scope of the present upgrade project) and are proposed to be sited in experimental Hall A.

Heavy photons, $\mathrm{A}^{\prime}$ s, are new hypothesized massive vector bosons that have a weak coupling to electrically charged matter, including electrons. The existence of an $\mathrm{A}^{\prime}$ could explain the discrepancy between the measured and observed anomalous magnetic moment of the muon ${ }^{11}$ and several intriguing dark matter-related anomalies. Three fixed-target experiments to search for these $\mathrm{A}^{\prime}$ are proposed using the high-quality and high-luminosity electron beam available at Jefferson lab: the $\mathrm{A}^{\prime}$ Experiment (APEX), ${ }^{12}$ the Heavy Photon Search (HPS), ${ }^{13}$ and Detecting $\mathrm{A}^{\prime}$ Resonance Kinematically with Electrons Incident on a Gaseous Hydrogen Target (Dark Light). ${ }^{14}$

\section{Electron Ion Collider (EIC)}

As discussed above in 3.2, the upgraded CEBAF at Jefferson Lab will provide unprecedented capability to study nucleon structure in the valence region where Bjorken $x>0.1$. However, there remains a crucial need to study the nucleon at lower $x<0.1$. Measurements in the 1990's at the HERA facility have shown that the parton dynamics at low $x$ is dominated by a large rise in the density of gluons. HERA collided electrons and protons at large center of mass energies, but it did not have polarized nucleon beams or nuclear beams, and the luminosity was constrained to be rather low $\left(\sim 10^{31} \mathrm{~cm}^{-2} \mathrm{~s}^{-1}\right.$. This limited luminosity is not sufficient to study nucleon tomography with GPD's and TMD's as will be possible at larger $x$ with the $12 \mathrm{GeV}$ CEBAF. A new Electron Ion Collider facility has been identified to address this important scientific issue. Recently, a white paper that addresses the scientific potential of the envisioned EIC facility has been produced. ${ }^{15}$ The nuclear physics QCD community has reached a consensus on the basic scientific requirements for such a facility:

- Highly polarized ( 70\%) electron and nucleon beams

- Ion beams from deuteron to the heaviest nuclei (Uranium or Lead)

- Variable center of mass energies from $\sim 20-\sim 100 \mathrm{GeV}$, upgradable to $\sim 150 \mathrm{GeV}$

- High collision luminosity $\sim 10^{33-34} \mathrm{~cm}^{-2} \mathrm{~s}^{-1}$.

Such a facility would capitalize on the powerful new experimental techniques for exploring nucleon structure that are being developed for the $12 \mathrm{GeV}$ JLab program, and apply them to the low $x$ region where the dynamics is dominated by the gluons. It is widely perceived that addressing this kinematic regime with high luminosity and fully polarized beams is necessary to complete our understanding of the basic 


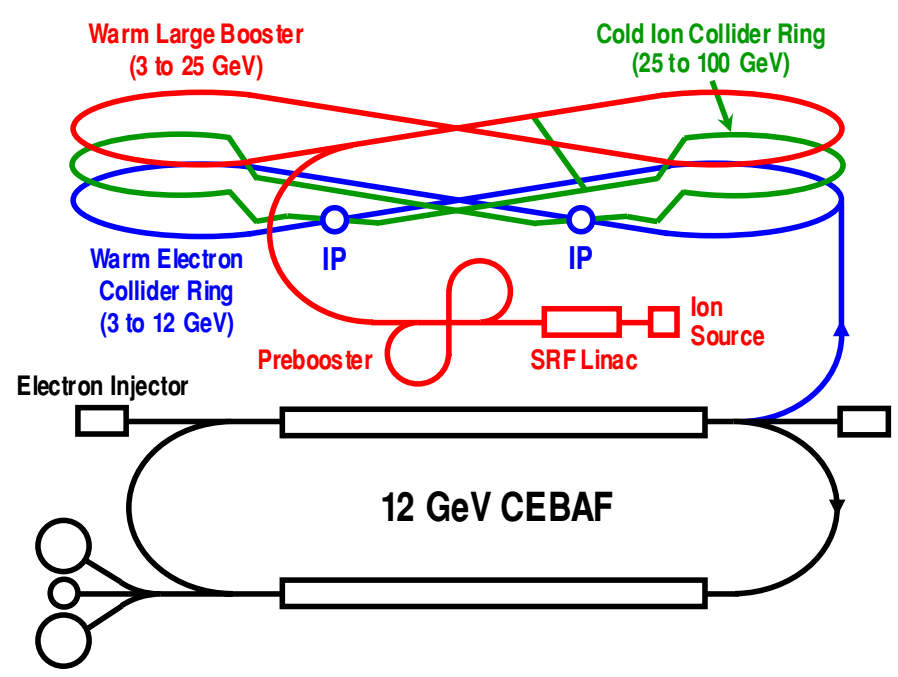

Fig. 2. A schematic drawing of MEIC.

partonic structure of the nucleon. An EIC is viewed as a natural extension of the capabilities of the Jefferson Lab $12 \mathrm{GeV}$ upgrade, the RHIC spin program, the HERMES experiment at DESY, and the COMPASS experiment at CERN.

The Accelerator and Physics Divisions at Jefferson Lab have developed a novel design for an EIC that would utilize the existing $12 \mathrm{GeV}$ CEBAF as an injector to a new collider facility. ${ }^{16}$ Shown in Fig. 2, the facility would employ storage rings in a "figure 8" layout to mitigate the effects of depolarizing resonances and facilitate high beam polarization. A medium energy version, MEIC, is envisioned as the first step, enabling collisions of $12 \mathrm{GeV}$ electrons with $100 \mathrm{GeV}$ protons. This could be later upgraded to the full EIC facility with $12 \mathrm{GeV}$ electrons colliding with $250 \mathrm{GeV}$ protons.

\section{Acknowledgments}

This material is based upon work supported by U.S. Department of Energy, Office of Science, Office of Nuclear Physics under contract DE-AC05-06OR23177.

\section{References}

1. D. Higinbotham, W. Melnitchouk, and A. W. Thomas, J. Phys., Volume 299 (2011).

2. J. Dudek, et al., Eur. Phys. J. A 48: 187 (2012).

3. J. J. Dudek, R. G. Edwards, B. Joo, M. J. Peardon, D. G. Richards and C. E. Thomas, Phys. Rev. D 83, 111502 (2011).

4. J. J. Dudek, R. G. Edwards, M. J. Peardon, D. G. Richards, and C. E. Thomas, Phys.Rev. D82 034508 (2010).

5. L.B. Weinstein et al., Phys. Rev. Lett. 106, 052301 (2011).

6. S. Abrahamyan, et al., Phys. Rev. Lett. 108, 112502 (2012). 
7. J. Beringer et al. (Particle Data Group), Phys. Rev. D86, 010001 (2012).

8. D. S. Armstrong, et al., Jefferson Lab proposal E08-016, (2007); http://www.jlab.org/ exp_prog/proposals/05/PR05-008.pdf

9. P. Bosted, et al., Jefferson Lab proposal E12-10-007, (2009); http://www.jlab.org/ exp_prog/proposals/10/PR12-10-007.pdf

10. J. Benesch et al., Jefferson Lab proposal E12-09-005. (2010); http://www.jlab.org/ exp_prog/proposals/09/PR12-09-005.pdf

11. G.W. Bennett et al., Phys. Rev. D 73072003 (2006).

12. J. D. Bjorken, et al., Jefferson Lab proposal E12-10-009 (2010).

13. A. Grillo, et al., Jefferson Lab proposal C12-11-006 (2012).

14. J. Balewski, et al., Jefferson Lab proposal C12-11-008 (2012).

15. A. Accardi, et al., arXiv:1212.1701 (2012).

16. S. Abeyratne, et al., arXiv:1209.0757 (2012). 